\title{
Using global transcription machinery engineering (gTME) to improve ethanol tolerance of Zymomonas mobilis
}

\section{Open Acces:} CrossMark

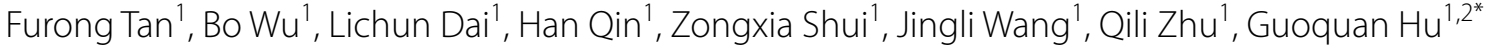 \\ and Mingxiong $\mathrm{He}^{1,2^{*}}$
}

\begin{abstract}
Background: With the increasing global crude oil crisis and resulting environmental concerns, the production of biofuels from renewable resources has become increasingly important. One of the major challenges faced during the process of biofuel production is the low tolerance of the microbial host towards increasing biofuel concentrations.

Results: Here, we demonstrate that the ethanol tolerance of Zymomonas mobilis can be greatly enhanced through the random mutagenesis of global transcription factor RpoD protein, $\left(\sigma^{70}\right)$. Using an enrichment screening, four mutants with elevated ethanol tolerance were isolated from error-prone PCR libraries. All mutants showed significant growth improvement in the presence of ethanol stress when compared to the control strain. After an ethanol (9\%) stress exposure lasting $22 \mathrm{~h}$, the rate of glucose consumption was approximately $1.77,1.78$ and $1.39 \mathrm{~g} \mathrm{~L}^{-1} \mathrm{~h}^{-1}$ in the best ethanol-tolerant strain ZM4-mrpoD4, its rebuilt mutant strain ZM4-imrpoD and the control strain, respectively. Our results indicated that both ZM4-mrpoD4 and ZM4-imrpoD consumed glucose at a faster rate after the initial $9 \%$ ( $\mathrm{v} / \mathrm{v}$ ) ethanol stress, as nearly $0.64 \%$ of the initial glucose remained after $54 \mathrm{~h}$ incubation versus approximately $5.43 \%$ for the control strain. At $9 \%$ ethanol stress, the net ethanol productions by ZM4-mrpoD4 and ZM4-imrpoD during the 30-54 $\mathrm{h}$ were 13.0-14.1 $\mathrm{g} / \mathrm{l}$ versus only $6.6-7.7 \mathrm{~g} / \mathrm{l}$ for the control strain. The pyruvate decarboxylase activity of ZM4-mrpoD4 was 62.23 and $68.42 \mathrm{U} / \mathrm{g}$ at 24 and $48 \mathrm{~h}$, respectively, which were 2.6 and 1.6 times higher than the control strain. After 24 and $48 \mathrm{~h}$ of $9 \%$ ethanol stress, the alcohol dehydrogenase activities of ZM4-mrpoD4 were also augmented, showing an approximate 1.4 and 1.3 times increase, respectively, when compared to the control strain. Subsequent quantitative real-time PCR analysis under these stress conditions revealed that the relative expression of pdc in cultured (6 and 24 h) ZM4-mrpoD4 increased by 9.0- and 12.7-fold when compared to control strain.
\end{abstract}

Conclusions: Collectively, these results demonstrate that the RpoD mutation can enhance ethanol tolerance in $Z$. mobilis. Our results also suggested that RpoD may play an important role in resisting high ethanol concentration in $Z$. mobilis and manipulating RpoD via global transcription machinery engineering (gTME) can provide an alternative and useful approach for strain improvement for complex phenotypes.

Keywords: Ethanol tolerance, Zymomonas mobilis, Random mutagenesis, Global transcription machinery engineering (gTME), Error-prone PCR, $\sigma^{70}$

\section{Background}

With the increasing global crude oil crisis and resulting environmental concerns, the production of biofuels from

\footnotetext{
*Correspondence: huguoquan1@hotmail.com; hemingxiong@caas.cn ${ }^{1}$ Biogas Institute of Ministry of Agriculture, Biomass Energy Technology Research Centre, Section 4-13, Renmin Nanlu, Chengdu 610041, China Full list of author information is available at the end of the article
}

renewable resources has become increasingly important [1]. To this end, bioethanol production has seen a sharp escalation over the past decades. In general, bioethanol can be produced by fermenting biological resources (e.g. energy-rich crops or lignocellulosic biomass) using a variety of potential microbes such as Saccharomyces cerevisiae, Escherichia coli, Klebsiella oxytoca, and 
Zymomonas mobilis [2]. Of these, Z. mobilis, a Gramnegative facultative anaerobic bacterium, has attracted considerable interest. Critically, it has a unique metabolism and ability to produce ethanol and/or other valuable chemicals from simple sugars via its unique Entner-Doudoroff (ED) pathway [3-6].

However, various environmental stressors can both adversely affect the microorganism growth of $Z$. mobiles and its ability to produce ethanol during fermentation. For instance, high ethanol concentrations, osmotic pressure, and oxidative stresses are all major stress that can impede the specific growth rate and viability of $Z$. mobilis cells as well as its ethanol production [7-9]. To better understand and address these limitations, it is essential to obtain mutant $Z$. mobilis strains that have improved stress tolerance [7, 10-14].

Past work has established that multi-gene regulation involving carbohydrate metabolism, cell membrane biogenesis, respiratory chain, DNA replication and recombination, transcriptional regulation, and some universal stress responses culminates in the stress tolerance of Z. mobilis [15-17]. Similarly, the genes associated with ethanol tolerance in yeast were also found to be linked to a broad range of different functional categories and biological functions [18, 19]. Recently, Henderson and Block (2014) also revealed that in S. cerevisiae, the lipid composition of the cellular membrane plays an important role in its response to ethanol stress [20]. Although many studies have been carried out to better understand the molecular basis of ethanol stress and tolerance in S. cerevisiae, it is still a challenging and difficult task to construct a wide enough variety of strains capable of responding to various stresses. The recent development of the global transcriptional engineering has attracted much attention in the field of strain engineering as a possible solution to this problem, particularly for those working on stress tolerance. Several transcription factors, including zinc finger-containing artificial transcription factor [21-23], sigma factor [24, 25], Spt15 [26], H-NS [27], Hha [28], and cAMP [29, 30], have been modified via global transcriptional engineering for improved strain tolerance and better control of biofilm formation. With this methodological development, a new route for identifying mutant transcription factor that can tolerate various inhibitors has been established. However, little work using global transcriptional engineering has focused on genetically improving the stress tolerance of $Z$. mobilis.

Since the RNA polymerase sigma subunit ( $\sigma$ factor) is known as a fundamental in promoter recognition and transcriptional initiation at the correct site, mutation of $\sigma$ factor might alter the promoter preferences of RNA polymerase. In turn, this could affect transcriptional levels, thus modulating the transcriptome on a global level. We thus sought to improve the ethanol tolerance of $Z$. mobilis ZM4 by engineering its rpoD gene, which encodes the main sigma factor, $\sigma^{70}$. The rpoD gene was subjected to error-prone PCR and cloned into a low-copy expression vector, pBBR1MCS-tet. Recombinant plasmids were then transformed into Z. mobilis ZM4 and random mutagenesis libraries were subjected to selection pressure using ethanol as a stress. Using this method, four error-prone PCR mutants with enhanced ethanol resistance were identified, all of which showed increased ethanol tolerance when compared to wild type. The mutant demonstrating the highest resistance, ZM4-mrpoD4, was subjected to further evaluation of its glucose utilization and key enzymatic activity. Finally, quantitative realtime PCR analysis was performed to detect the expression levels of several genes related to $Z$. mobilis metabolic pathways.

\section{Methods}

\section{Materials}

E. coli $\mathrm{DH} 5 \alpha$ was cultured in LB medium and used as the host organism for all common transformations. Plasmid pBBR1MCS-tet was derived from pBBR1MCS [31]. Restriction enzymes were purchased from Fermentas (Burlington, Canada). E.Z.N.A. ${ }^{\circledR}$ Gel Extraction Kit and E.Z.N.A. Plasmid Mini Kit I were obtained from Omega Bio-Tek (Norcross, GA, USA). T4 DNA ligase was obtained from Thermo Scientific (Ipswich, MA, USA) and was used for ligations. GeneMorph ${ }^{\circledR}$ II Random Mutagenesis Kit was obtained from Stratagene (La Jolla, CA, USA). HotMaster Taq DNA polymerase was obtained from Tiangen Biotech (Beijing, China). The primers used in this study are summarized in Table 1.

\section{Construction of random mutagenesis libraries}

Error-prone PCR was performed using $180 \mathrm{ng}$ of rpoD. Resulting PCR products were then subjected to the GeneMorph II Random Mutagenesis Kit (Stratagene) using various concentrations of initial template. This approach yielded low (0-4.5 mutations/kb), medium (4.5-9 mutations/kb), and/or high mutation (9-16 mutations/kb) rates as described in the manufacturer's protocol. Following PCR, fragments were purified using E.Z.N.A. ${ }^{\circledR}$ Gel Extraction Kit (Norcross, GA, USA) according to the manufacturer's instructions, digested by Xho I and Xba $\mathrm{I}$, and ligated into the corresponding restriction sites of pBBR1MCS-tet, which contained the pyruvate decarboxylase (PDC) promoter and terminator in order to generate either the recombinant plasmid PBmrpoD or PBrpoD (harboring the unmutated version of $r p o D$ gene) (Fig. 1). Plasmids were then transformed into Z. mobilis 
Table 1 Primer sequences with restriction site underlined

\begin{tabular}{ll}
\hline Primer & Sequence \\
\hline Ppdc Kpnl F & 5'-CGG GGTACCTTACGCTCATGATCGC-3' \\
Ppdc Xhol R & 5'-CCG CTCGAG TGCTTACTCCATATAT-3' \\
1623 Xhol F & 5'-CCG CTCGAG ATGGCAGAGACGACTACGG-3' \\
1623 Xbal R & 5'-TGCTCTAGACTAGTGGTCGAGGAAGCTCC-3' \\
Tpdc Xbal F & 5'-GACGGCTCTAGATAGTTTTTAAATAAACTTAGAGCT \\
& TAAG-3' \\
Tpdc Sacl R & 5'-GCCTCGAGCTCAATTTTATAGAAAAGAAAAACAAAGC-3' \\
ZMO1360 F & 5'-TACAACCTCGTCCTTCTT-3' \\
ZMO1360 R & 5'-CATAACCTTCTGCACTGA-3' \\
ZMO1596 F & 5'-GGTATTAATTCTGCTGTT-3' \\
ZMO1596 R & 5'-CGAAGTCTGAATTGTTAT-3' \\
16s F & 5'-TCAACTATAGACCAGTAAGT-3' \\
$16 s$ R & 5'-AGAACATAGAAGAGGTAAGT-3' \\
\hline &
\end{tabular}

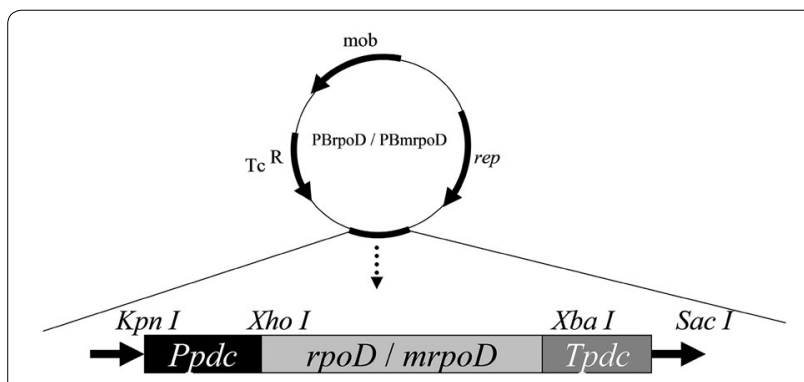

Fig. 1 Schematic illustration of recombinant plasmids $p B r p o D$ or pBmrpoD. Ppdc and Tpdc indicate the promoter and terminator of pyruvate decarboxylase, respectively

ZM4 via electroporation, after which cells were plated on RM-agar plates containing $5 \mu \mathrm{g} / \mathrm{ml}$ of tetracycline for culturing 4-5 day and scraped off to create a liquid library.

\section{Phenotype selection}

Transformants were incubated in $5 \mathrm{ml} \mathrm{RM}$ medium at $30{ }^{\circ} \mathrm{C}$ without shaking. One percent of the overnight cell culture was then inoculated into fresh RM supplemented with increasing initial ethanol concentration $(7,8$, and $9 \%(\mathrm{v} / \mathrm{v})$, sequentially) for $24 \mathrm{~h}$. After three rounds of selection, cells were spread onto RM-agar plates containing $5 \mu \mathrm{g} / \mathrm{ml}$ of tetracycline and the ethanol (9\%) stress. Individual colonies were randomly selected, plasmids extracted, and mutations verified using DNA sequencing. All mutant strains were compared to control strains $Z$. mobilis ZM4 and ZM4-rpoD, which harbored the recombinant plasmid PBrpoD as described above. By using controls, the influence of the plasmid and/or any interference between the plasmid and chromosomal copies of $r p o D$ were neutralized.

\section{Cell growth profiling}

To generate growth curves for both the mutant and control strains, cells were cultivated in a Bioscreen $\mathrm{C}$ system (Lab Systerms Helsinki, Finland) according to the manufacturer's instructions. Briefly, 1:10 of the overnight seed $(\mathrm{v} / \mathrm{v})$ was inoculated into $1 \mathrm{ml}$ fresh $\mathrm{RM}$ medium containing a range of initial ethanol concentrations $[0,6,8$ and $10 \%(\mathrm{v} / \mathrm{v})]$ with a similar initial $\mathrm{OD}_{600}$ value between 0.15-0.2. Cells were then added in triplicate into the wells of the Bioscreen plate with an end working volume of $300 \mu \mathrm{l} /$ well. Temperature was controlled at $30^{\circ} \mathrm{C}$ and the $\mathrm{OD}$ at $600 \mathrm{~nm}$. Absorbance values of the cell suspensions were automatically read at regular intervals of $1 \mathrm{~h}$ over a $48 \mathrm{~h}$ time period. Before each measurement, cell cultures were automatically shaken for $60 \mathrm{~s}$ to homogenize the samples.

\section{Glucose utilization and ethanol analysis under ethanol stress condition}

The mutant rpoD gene from the best ethanol-tolerant strain was cloned back to freshly prepared pBBR1MCStet plasmids described above, back-transformed into wild type strain ZM4 to proof that only this mutation is responsible for phenotype. Cells were grown in $\mathrm{RM}$ medium containing $20 \mathrm{~g} / \mathrm{l}$ glucose at $30{ }^{\circ} \mathrm{C}$ to the mid$\log$ phase. Ten $\mathrm{ml}$ of the culture was then transferred into $100 \mathrm{ml}$ fresh RM medium (50 g/l glucose) containing $9 \%(\mathrm{v} / \mathrm{v})$ ethanol with a initial $\mathrm{OD}_{600}$ value of approximately 0.2 . Cells were grown at $30{ }^{\circ} \mathrm{C}$ for $2-3$ days. The $\mathrm{OD}_{600}$ was monitored by measuring the optical density at $600 \mathrm{~nm}$ with a UV765 spectrophotometer. Glucose and ethanol were measured using high performance liquid chromatography (HPLC) (Agilent Hi-plex H, $300 \times 7.7 \mathrm{~mm})$ with sulfuric acid $(0.05 \mathrm{M})$ as the mobile phase, a flow rate of $0.6 \mathrm{~mL} / \mathrm{min}$, and a column temperature of $35^{\circ} \mathrm{C}$. The net ethanol production was calculated by the total ethanol minus the initial $9 \%$ ethanol.

\section{Quantitative-PCR analysis}

Total RNA was isolated using Trizol reagent and the resulting RNA samples were reverse-transcribed using the QuantScript RT Kit (TIANGEN, Beijing, China) as described in the manufacturer's protocol. The expression of representative identified genes $(a d h B$ and $p d c)$ from different treatments were quantified by quantitative realtime PCR (qPCR) using a BIO-RAD Real-Time PCR-iQ5 System. All optimized primers are shown in Table 1 and were designed using primer software to amplify approximately $100 \mathrm{bp}$ from the $3^{\prime}$ end of the target genes. PCR conditions were $15 \mathrm{~min}$ at $94{ }^{\circ} \mathrm{C}$, followed by 40 cycles of heating at $94{ }^{\circ} \mathrm{C}$ for $20 \mathrm{~s}$ and $50-55{ }^{\circ} \mathrm{C}$ for $30 \mathrm{~s}$, and $72{ }^{\circ} \mathrm{C}$ for $20 \mathrm{~s}$, and final extension at $72{ }^{\circ} \mathrm{C}$ for $5 \mathrm{~min}$. PCR amplification was detected by SYBR Green (TIANGEN, 
Beijing, China). The ratios of the cycle threshold $(\mathrm{Ct})$ values were determined from the included BIO-RAD iQ5 Optical System Software. The $\Delta \Delta \mathrm{Ct}$ method was chosen to analyze gene expression levels and standard curves for each primer were plotted to ensure similar amplification efficiency when compared with the reference gene. The rrsA gene, encoding the 16S RNA, served as an endogenous control to normalize for differences in total RNA quantity.

\section{Enzyme assay}

Pyruvate decarboxylase (PDC) activity was determined by a previously described method [32] by monitoring the pyruvicacid-dependent oxidation of NADH with ADH as a coupling enzyme at $\mathrm{pH} 6.5$. The reaction was carried out at $25^{\circ} \mathrm{C}$ in $50 \mathrm{mM}$ sodium citrate buffer (pH6.5) containing $0.15 \mathrm{mM}$ NADH, $5 \mathrm{mM} \mathrm{MgCl}, 0.1 \mathrm{mM}$ TPP, $5 \mathrm{mM}$ pyruvate, and $10 \mu \mathrm{l}(10 \mathrm{U})$ of $\mathrm{ADH}$. The reaction was started by the addition of $10 \mu \mathrm{l}$ of cell-free extract. The rate of NADH oxidation was measured at $340 \mathrm{~nm}$.

Alcohol dehydrogenase (ADH) activity was assayed by measuring the alcohol-dependent reduction of $\mathrm{NAD}^{+}$at $\mathrm{pH}$ 6.5. Cells were permeabilized using methods designed for enzymatic assays as previously described [33, 34]. Cell lysates $(10-30 \mu \mathrm{l})$ were added for a final volume of $1 \mathrm{ml}$ (333 mM ethanol, $8.3 \mathrm{mM} \mathrm{NAD}^{+}$in $50 \mathrm{mM}$ sodium phosphate buffer, $\mathrm{pH}$ 6.5). The production of NADH was assayed from the change in absorbance at $340 \mathrm{~nm}$. One unit of PDC/ADH activity was defined as the generation of $1 \mu \mathrm{mol} \mathrm{NAD}{ }^{+} / \mathrm{NADH}$ per minute under the conditions specified. Enzyme activities were reported as international units per milligram of total cell protein. Protein was measured by the Lowry method with bovine serum albumin as a standard.

\section{Results and discussion}

Isolation of ethanol-tolerant RpoD mutants

Recombinant plasmids PBmrpoD were transformed into competent $Z$. mobilis ZM4 and the subsequent mutagenesis libraries were tested in subcultures with repeated ethanol [7, 8 and $9 \%(\mathrm{v} / \mathrm{v})]$ administration to separate those successful mutants harboring enhanced ethanol tolerance. Cells were spread onto RM-agar plates containing $5 \mu \mathrm{g} / \mathrm{ml}$ of tetracycline and the initial ethanol (9 \%) stress. Using this method, approximately several dozen strains were initially screened from the RM-agar plates. Of these, four ethanol-tolerant mutant strains (ZM4-mrpoD1, ZM4-mrpoD2, ZM4-mrpoD3 and ZM4mrpoD4) that had markedly better cell growth under ethanol stress were selected for further analysis.

These four mutants were compared in growth performance between ZM4 and ZM4-rpoD. Initial studies of growth characteristics of strains in the presence of initial ethanol $7 \%(\mathrm{v} / \mathrm{v})$ indicated that both this control strain and an alternative control containing only a blank plasmid (no rpoD gene) had similar growth rates. As a result, we chose to use the strain ZM4-rpoD as the sole control strain for all further experiments presented here.

\section{RpoD mutant growth}

We then sought to investigate the effects of ethanol stress on the growth of the RpoD mutant and control strains. The resulting growth curves are presented in Fig. 2. The ethanol tolerances of the four ethanol-tolerant mutants were investigated at different initial ethanol [6, 8 and $10 \%$ $(\mathrm{v} / \mathrm{v})$ ] concentrations by comparing their growth performance to that of the control. When cultured without initial ethanol addition, all mutants and control presented similar cell growth curve (Fig. 2). As the initial ethanol concentration increased in the culture medium, all mutants showed better growth than control, with mutant ZM4-mrpoD4 displaying the best ethanol tolerance of the four. As shown in Fig. 2, in the presence of $6 \%$ ethanol stress, ZM4-mrpoD4 entered the plateau phase after 7-8 h, which was significantly ahead ( $3 \mathrm{~h}$ ) of the control strain. When initial ethanol concentration reached $8 \%$ (v/v), ZM4-mrpoD4 reached its highest cell density of 0.9 $\left(\mathrm{OD}_{600}\right)$, while that of control strain was 0.4. When the initial ethanol concentration was increased to $10 \%(\mathrm{v} / \mathrm{v})$, all strains growth slowed, but mutant growth remained much faster than that of control.

\section{Effect of ethanol stress on glucose utilization and ethanol production}

Since the mutant ZM4-mrpoD4 had demonstrated the best ethanol resistance among all four mutants, its mutant $r p o D$ gene was cloned back to freshly prepared pBBR1MCS-tet plasmids and back-transformed into wild type strain ZM4 to create strain ZM4-imrpoD. During ethanol fermentation, ethanol stress may adversely affect the ability of the cell to perform efficient and consistent conversion of sugars to ethanol. Given this, we sought to examine the influence of the RpoD mutation on the fermentation ability of $Z$. mobilis (Fig. 3). From the Fig. 3, there were no differences between mutant strains (ZM4-mrpoD4 and ZM4-improD) and control strain ZM4-rpoD in terms of the growth, glucose utilization, and ethanol yield at normal condition. However, in the RM medium containing ethanol (9 \%, v/v) stress, ZM4mrpoD4 and ZM4-imrpoD reached their maximal cell density $\left(\mathrm{OD}_{600}\right)$ at approximately 1.8 after the initial $30 \mathrm{~h}$ incubation. Comparatively, the control strain reached its highest cell density of approximately $1.2\left(\mathrm{OD}_{600}\right)$ under the same condition. After $22 \mathrm{~h}$ of the ethanol (9\%) stress, the rate of glucose consumption was about 1.77, 1.78 and $1.39 \mathrm{~g} \mathrm{~L}^{-1} \mathrm{~h}^{-1}$ in ZM4-mrpoD4, ZM4-imrpoD and 

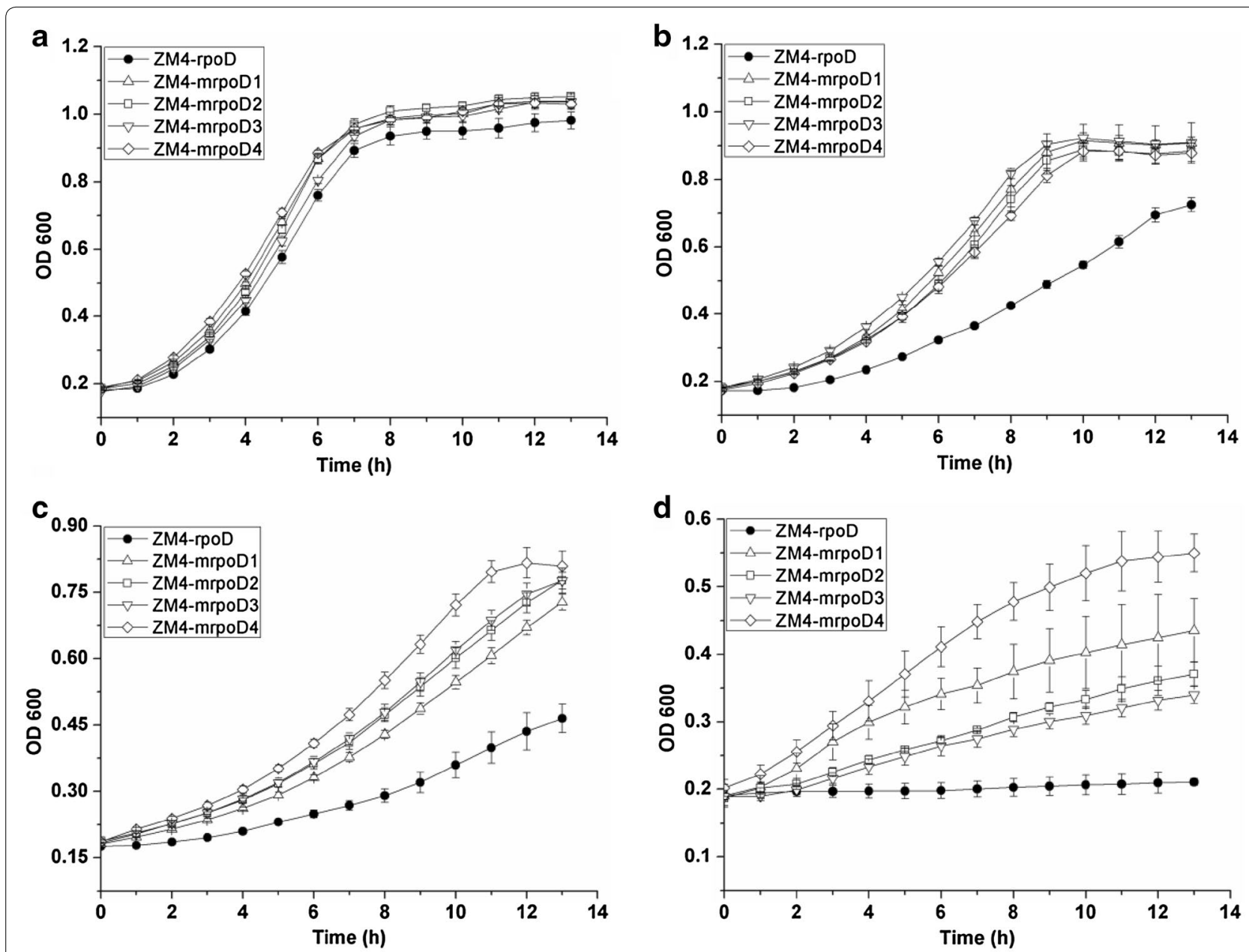

Fig. 2 Growth of RpoD mutants and control strain ZM4-rpoD in RM medium. Control strain ZM4-rpoD contains the un-mutated version of rpoD gene, cells were grown in a $0 \%$ ethanol, b $6 \%$ ethanol, c $8 \%$ ethanol and d $10 \%(\mathrm{v} / \mathrm{v})$ ethanol stress at $30^{\circ} \mathrm{C}$

control strain, respectively. These data clearly indicated that ZM4-mrpoD4 and ZM4-imrpoD consumed glucose faster under ethanol stress conditions, as nearly $18 \%$ of the initial glucose remaining after $22 \mathrm{~h}$ incubation, versus about $36 \%$ for the control strain. When fermented for $54 \mathrm{~h}$ in the presence of ethanol $(9 \%, \mathrm{v} / \mathrm{v})$, the initial glucose remained in the cultures of the control strain and mutants strains was approximately 5.43 and $0.64 \%$, respectively. We also measured the net ethanol productions of mutant strains and control strain under the process of fermentation at normal condition and $9 \%$ ethanol stress. Our results indicated that the net ethanol production by ZM4-mrpoD4 and ZM4-imrpoD during the $30-54 \mathrm{~h}$ were $13.0-14.1 \mathrm{~g} / \mathrm{l}$ versus only $6.6-7.7 \mathrm{~g} / \mathrm{l}$ for the control strain, thus indicating that ZM4-mrpoD4 and ZM4-imrpoD can produce more ethanol than the control strain under the condition of $9 \%$ ethanol stress, which was consistent with its higher cell growth and faster glucose consumption under the ethanol stress condition.
We speculate that the ethanol tolerance of mutant strain may be due to some stress response mechanism. In the mutant strain, the expression level of some stress-responsive genes may be increased after exposure to ethanol. Therefore, further studies including transcriptomics and metabolomics are required to clarify its tolerance mechanism of RpoD mutation in conferring improved ethanol tolerance in Z. mobilis.

\section{Effects of ethanol stress on enzymatic activities}

For normal physiological operation of the Entner-Doudoroff (ED) pathway, both PDC and ADH key enzymes are required in $Z$. mobilis. Given this importance, the PDC and ADH activities of ZM4-mrpoD4 and the corresponding control strain were compared under the initial ethanol (9\%) stress after incubations lasting 6, 24, and $48 \mathrm{~h}$. The PDC and ADH activities of ZM4-mrpoD4 and the control strain were not significantly different at $6 \mathrm{~h}$, while the activities of the two enzymes from 

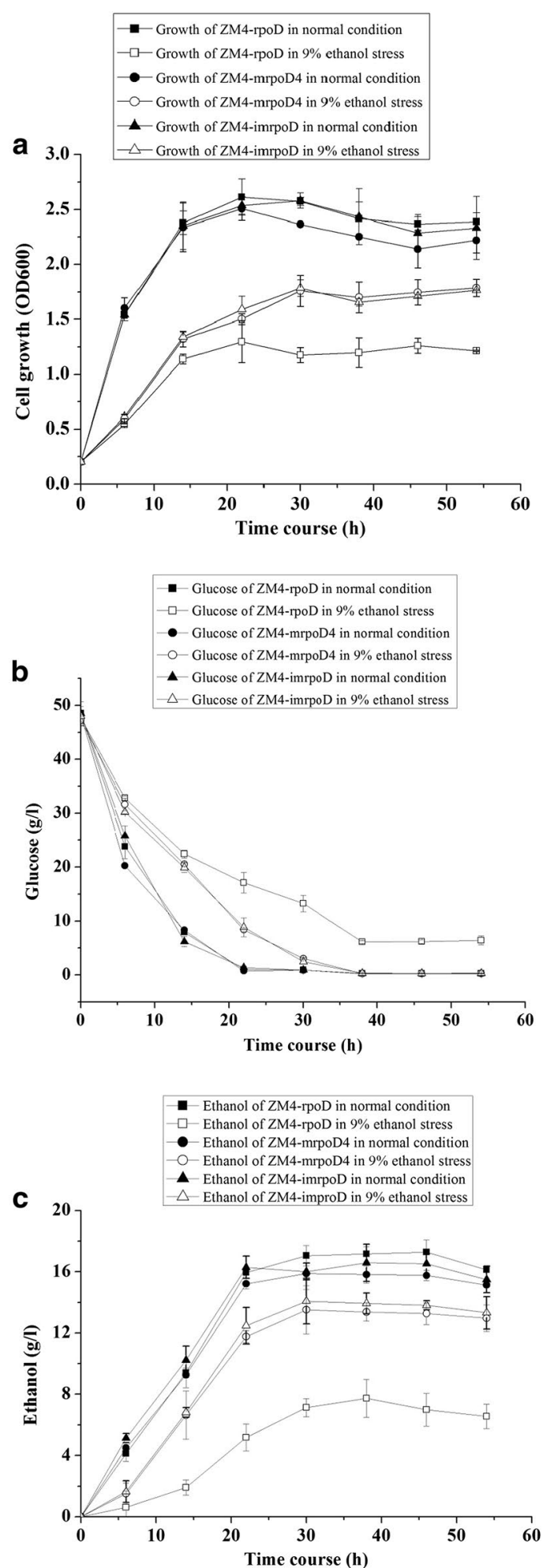

Fig. 3 Effects of ethanol stress on growth, glucose utilization, and ethanol yield of mutant strains and control strain. Cells were grown in normal condition and $9 \%$ ethanol stress, data are presented as the mean values of samples run in triplicate. a cell growth $\left(\mathrm{OD}_{600}\right)$; b glucose concentration ( $\mathrm{g} / \mathrm{l})$; c ethanol (g/l)
ZM4-mrpoD4 were significantly higher than those of the control strain at both $24 \mathrm{~h}$ and $48 \mathrm{~h}$ (Fig. 4). To this end, the PDC activity of ZM4-mrpoD4 was 62.23 and $68.42 \mathrm{U} / \mathrm{g}$ at 24 and $48 \mathrm{~h}$ under the initial ethanol (9\%) stress, respectively. These results show an increase of 2.6 and 1.6 times over that of the control strain. Similarly, the ADH activity of ZM4-mrpoD4 was also enhanced under conditions of the initial ethanol (9\%) stress, revealing increases of 1.4 and 1.3 times as that of control strain at 24 and $48 \mathrm{~h}$.

In addition, we measured transcription levels of $a d h B$ and $p d c$ through the use of quantitative RT-PCR. In the absence of ethanol stress, our results showed that the expression level of the $a d h B$ gene in ZM4-mrpoD4 cultured for $6 \mathrm{~h}$ did not show differential expression from control strain. However, the $p d c$ gene was up-regulated by about 2.2-fold $(p>0.05)$. The expression levels of the $a d h B$ and $p d c$ genes in a $24 \mathrm{~h}$ ZM4-mrpoD4 culture were downand up-regulated by about 0.6 - and 2.7-fold $(p>0.01)$, respectively (Fig. 5). When the cells were exposed to the initial ethanol (9\%) stress for $6 \mathrm{~h}$, the expression level of $a d h B$ in ZM4-mrpoD4 was not statistically different from that of control strain. In contrast, ZM4-mrpoD4 cultured for $24 \mathrm{~h}$ had $a d h B$ levels down-regulated by about 0.5 -fold $(p>0.01)$. Interestingly, the level of $p d c$ mRNA in ZM4mrpoD4 cultured for either 6 or $24 \mathrm{~h}$ increased by 9.0 and 12.7 -fold, respectively, when compared to the control strain $(p>0.01)$ (Fig. 5). We should note that in our earlier global profiling study using $5 \%$ ethanol stress, the expression levels of $p d c$ and $a d h B$ were down-regulated by about 0.8 - and 0.9-fold, respectively [16].

\section{Sequence alignment and mutational analysis of the mutants}

Mutant $r p o D$ genes were also sequenced using the primers 1623 XhoI F and 1623 XbaI R (Table 1). Sequences were aligned and compared using Clustal $\mathrm{W}$ version 2.0. Their amino acid substitutions are summarized in Table 2 and Fig. 6. As shown in Table 2 and Fig. 6, 13 total point mutations (Q57L, G97S, P195T, D203V, D206E, R324H, M369L, E370D, G426C, I448N, E573G, A592V and L606S) were adopted by these RpoD mutants to cope with the ethanol stress. Among them, three point mutations (R324H, M369L and E370D) are located at non-essential regions, making their function unknown, but allowing for their removal without a corresponding loss of function. Two point mutations (E573G and A592V) fell into conserved region 3, and three substitutions (Q57L, I448N, and L606S) were present in regions $1.1,2$, and 4 , respectively. Our current hypothesis is that these mutations exert differential effects on promoter recognition and transcription initiation. 

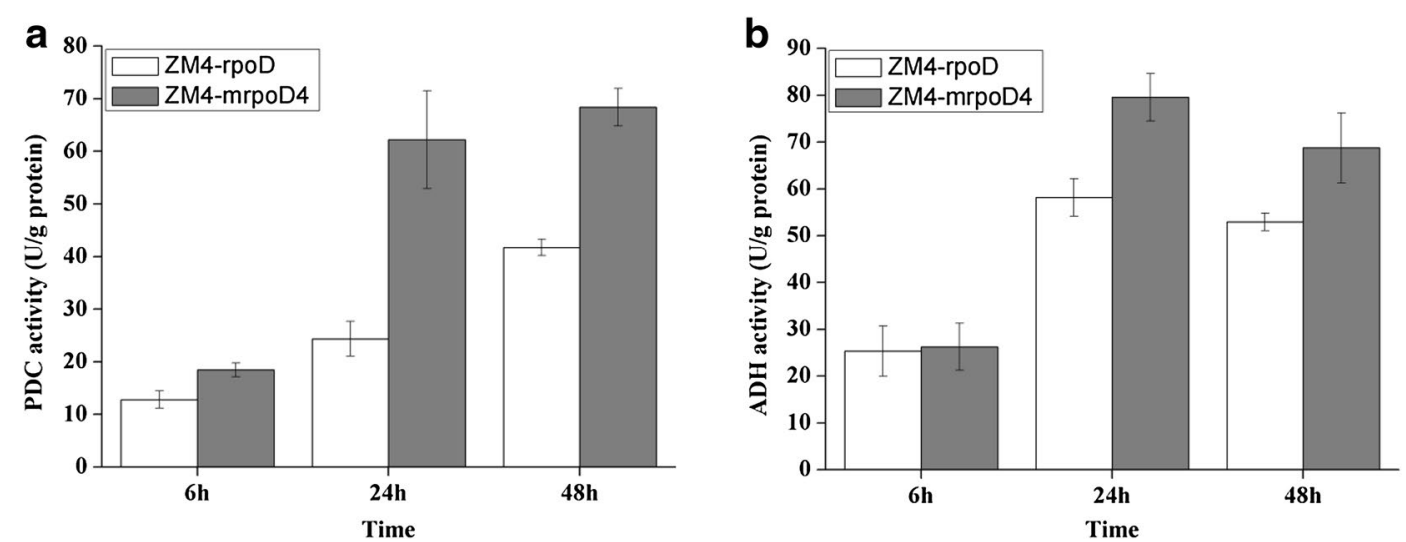

Fig. 4 Pyruvate decarboxylase (PDC) and alcohol dehydrogenase (ADH) activities of crude extracts of ZM4-mrpoD4 and control strain under ethanol stress condition

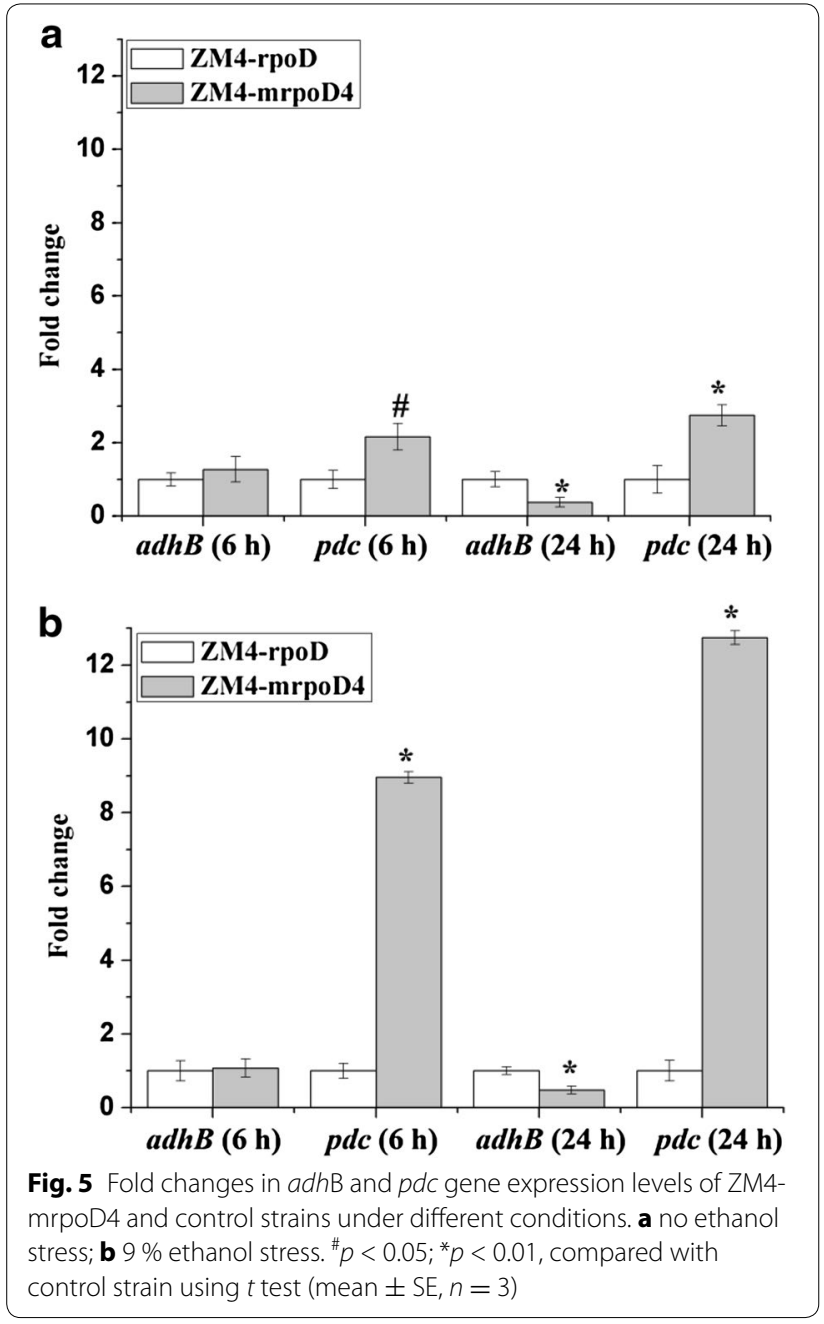

Table 2 Amino acid substitutions in four mutant strains

\begin{tabular}{ll}
\hline Mutants & Amino acid substitution \\
\hline ZM4-mrpoD1 & P195T \\
ZM4-mrpoD2 & D203V R324H A592V L606S \\
ZM4-mrpoD3 & G97S D206E M369L E370D E573G \\
ZM4-mrpoD4 & Q57L G426C 1448N \\
\hline
\end{tabular}

RpoD is an RNA polymerase sigma subunit composed of N-terminal domain of region 1.1 (residues 18-88) and region 1.2 (residues 116-149), non-essential region (residues 245-405) and C-terminal domain of region 2 (residues 437-507), region 3 (residues 516-593) and region 4 (residues 599-657) (NCBI conserved domain 2015) (Fig. 6). Analysis of the mutations found in the four mutants revealed several interesting features. First, we found that simple modification to the sigma factor RpoD led to enhanced strain tolerance towards ethanol stress. Second, the mutations occurred in all four of the predicted conserved regions, with the exception of mutant ZM4-mrpoD1, whereby only one mutation was found in P195T and did not include in any of the conserved regions (Fig. 6). Furthermore, though some of mutations are located in the conserved regions of the protein, none of the DNA binding residues (T610, R620, T629, L630, T641, E643, R644, R646, Q647, I648, A650, K651 and L653) on conserved domain region 4 were mutated.

The mutant ZM4-mrpoD4 with the highest ethanol tolerance had three mutations present (Q57L, G426C and $\mathrm{I} 448 \mathrm{~N})$. Residue Q57 is one of the residues of region 1.1, which is known to be responsible for modulating DNA and promoter binding to allow for proper transcription 


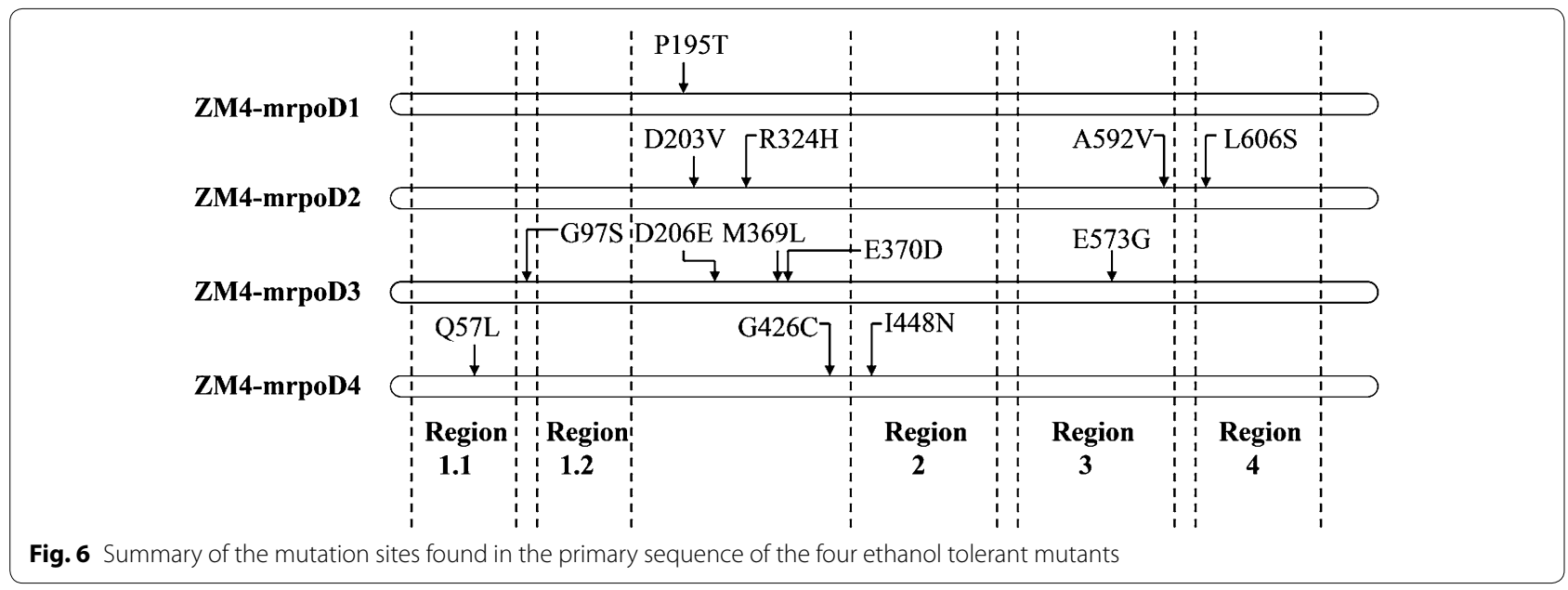

initiation [35]. Given this role, it is possibly that mutation Q57L could influence DNA and promoter binding to RNA polymerase. Residue I448 lies in region 2, which contains both the -10 promoter recognition helix and the primary core RNA polymerase binding determinant [35]. It is therefore possible that point mutation I448N may also impact transcription through currently unknown mechanism. However, the structure-function relationship between these mutations and the observed ethanol tolerances remains unclear and in need to future investigation. To this end, further studies are required to identify its direct target genes and/or interacting partners to better elucidate the molecular mechanisms behind how mutations in RpoD can confer improved ethanol stress tolerance in Z. mobilis. Moreover, it will be interesting to ascertain the global transcriptional differences in strains harboring the mutation to ultimately identify the gene expression changes resulting in enhanced ethanol tolerance.

Having the sequenced genome of Z. mobilis ZM4 allows for better efforts at strain development [36]. In our previous study, our lab successfully used microarray technology to investigate expression profiling of the ethanologenic $Z$. mobilis ZM4 in response to ethanol stress [16]. Our results showed 127 genes were either up- or down-regulated in response to ethanol stress. Among these, sigma factors-those responsible for stress tolerance in $E$. coli-were also shown to be highly differential in their expression. These included sigma-E $\left(\sigma^{E}\right.$, ZMO1404, 1.3-fold), $\sigma^{70}$ (rpoD, ZMO1623, 1.7-fold), $\sigma^{54}$ (rpoN, ZMO0274, 1.2-fold), and $\sigma^{28}$ (fliA, ZMO0626, 1.4-fold). Seo et al. [36] supposed that sigma-E plays a key role in resisting high ethanol condition in $Z$. mobilis, which is in keeping with our current results . In further support, Palonen et al. [37] also suggested that sigma-E is significantly involved in the stress tolerance of Yersinia pseudotuberculosis IP32953 . In the present study, RpoD mutation augmented $Z$. mobilis ethanol tolerance. Our results suggest that sigma 70 may also play an important role in resisting high ethanol concentration in Z. mobilis, with manipulation of $\sigma^{70}$ allowing for another avenue for strain improvement.

\section{Conclusions}

The present study used global transcriptional engineering tools to enhance the ethanol tolerance of $\mathrm{Z}$. mobilis by rewiring its global regulator, RpoD. Mutations were introduced into RpoD via error-prone PCR and an enrichment screening procedure to isolate RpoD variants with enhanced ethanol resistance. Four mutants with enhanced ethanol tolerance were identified from error-prone PCR libraries. All mutants exhibited much better tolerance towards ethanol stress. Both the best ethanol-tolerant strain ZM4-mrpoD4 and its rebuilt mutant strain ZM4-imrpoD consumed glucose faster and produced more ethanol under ethanol stress conditions when compared to the control strain. Methodologically, our results further suggest that global transcription machinery engineering (gTME) is a viable route for strain engineering aimed at improving the complex phenotypes in Z. mobilis.

\section{Abbreviations}

ED: Entner-Doudoroff; PDC: pyruvate decarboxylase; ADH: alcohol dehydrogenase; gTME: global transcription machinery engineering; HPLC: high performance liquid chromatography.

\section{Authors' contributions}

FRT carried out all of the experiments, participated in the study design and wrote the draft manuscript. MXH designed the whole experiments and contributed to edit the draft manuscript. ZXS, HQ and JLW performed the profiling of fermentation. BW, LCD and GQH participated in discussion and helped in draft manuscript editing. All authors read and approved the final manuscript. 


\section{Author details}

${ }^{1}$ Biogas Institute of Ministry of Agriculture, Biomass Energy Technology Research Centre, Section 4-13, Renmin Nanlu, Chengdu 610041, China. ${ }^{2}$ Key Laboratory of Development and Application of Rural Renewable Energy, Ministry of Agriculture, Chengdu 610041, China.

\section{Acknowledgements}

This work was supported by Applied Basic Research Programs of Sichuan province (NO. 2014JY0065). Partially supported by the National Natural Science Foundation of China (Grant No.31570055), Youth Science and Technology Foundation of Sichuan Province (2015JQ0047), Open Funds of State Key Laboratory of Microbial Technology (Shandong University, M2013-07), Open Funds of Key Laboratory of Microbial Resources Collection and Preservation (Ministry of Agriculture, MOA, 2013), Open Funds of Xinjiang Production \& Construction Corps Key Laboratory of Protection and Utilization of Biological Resources in Tarim Basin (Tarim University, BRZD1403).

\section{Competing interests}

The authors declare that they have no competing interests.

Received: 28 July 2015 Accepted: 15 December 2015

Published online: 13 January 2016

\section{References}

1. Luque R, Herrero-Davila L, Campelo JM, Clark JH, Hidalgo JM, Luna $D$, et al. Biofuels: a technological perspective. Energ Environ Sci. 2008;1(5):542-64.

2. Rubin EM. Genomics of cellulosic biofuels. Nature. 2008;454(7206):841-5.

3. dos Santos Dda S, Camelo AC, Rodrigues KC, Carlos LC, Pereira N Jr. Ethanol production from sugarcane bagasse by Zymomonas mobilis using simultaneous saccharification and fermentation (SSF) process. Appl Biochem Biotechnol. 2010;161(1-8):93-105.

4. He MX, Wu B, Qin H, Ruan ZY, Tan FR, Wang JL, et al. Zymomonas mobilis: a novel platform for future biorefineries. Biotechnol Biofuels. 2014;7(1):101-15.

5. Jeon YJ, Xun Z, Rogers PL. Comparative evaluations of cellulosic raw materials for second generation bioethanol production. Lett Appl Microbiol. 2010:51(5):518-24.

6. Rogers PL, Jeon YJ, Lee KJ, Lawford HG. Zymomonas mobilis for fuel ethanol and higher value products. Adv Biochem Eng Biotechnol. 2007; 108:263-88.

7. Franden MA, Pilath HM, Mohagheghi A, Pienkos PT, Zhang M. Inhibition of growth of Zymomonas mobilis by model compounds found in lignocellulosic. Biotechnol Biofuels. 2013;6:99-113.

8. Michel GP, Starka J. Effect of ethanol and heat on the protein pattern of Zymomonas mobilis. J Bacteriol. 1986;165(3):1040-2.

9. Thanonkeo P, Laopaiboon P, Sootsuwan K, Yamada M. Magnesium ions improve growth and ethanol production of Zymomonas mobilis under heat or ethanol stress. Biotechnology. 2007;6(1):112-9.

10. Chen M. irrE, an exogenous gene from Deinococcus radiodurans, improves the growth of and ethanol production by a Zymomonas mobilis strain under ethanol and acid stresses. J Microbiol Biotechnol. 2010;20(7):1156-62

11. Luo LH, Seo PS, Seo JW, Heo SY, Kim DH, Kim CH. Improved ethanol tolerance in Escherichia coli by changing the cellular fatty acids composition through genetic manipulation. Biotechnol Lett. 2009;31(12):1867-71.

12. Shi DJ, Wang CL, Wang KM. Genome shuffling to improve thermotolerance, ethanol tolerance and ethanol productivity of Saccharomyces cerevisiae. J Ind Microbiol Biotechnol. 2009;36(1):139-47.

13. Tao F, Miao JY, Shi GY, Zhang KC. Ethanol fermentation by an acid-tolerant Zymomonas mobilis under non-sterilized condition. Process Biochem. 2005;40(1):183-7.

14. Yang S, Land ML, Klingeman DM, Pelletier DA, Lu TY, Martin SL, et al. Paradigm for industrial strain improvement identifies sodium acetate tolerance loci in Zymomonas mobilis and Saccharomyces cerevisiae. Proc Natl Acad Sci USA. 2010;107(23):10395-400.

15. He MX, Wu B, Shui ZX, Hu QC, Wang WG, Tan FR, et al. Transcriptome profiling of Zymomonas mobilis under furfural stress. Appl Microbiol Biotechnol. 2012;95(1):189-99.
16. He MX, Wu B, Shui ZX, Hu QC, Wang WG, Tan FR, et al. Transcriptome profiling of Zymomonas mobilis under ethonal stress. Biotechnol Biofuels. 2012;5:75-84

17. Yang S, Pan C, Tschaplinski TJ, Hurst GB, Engle NL, Zhou W, et al. Systems biology analysis of Zymomonas mobilis ZM4 ethanol stress responses. PLoS One. 2013;8(7):68886-99.

18. Kasavi C, Eraslan S, Arga KY, Oner ET, Kirdar B. A system based network approach to ethanol tolerance in Saccharomyces cerevisiae. BMC Syst Biol. 2014:8(1):90-103.

19. Lewis JA, Broman AT, Will J, Gasch AP. Genetic Architecture of ethanolresponsive transcriptome variation in Saccharomyces cerevisiae strains. Genetics. 2014;198(1):369-82.

20. Henderson CM, Block DE. Examining the role of membrane lipid composition in determining the ethanol tolerance of Saccharomyces cerevisiae. Appl Environ Microbiol. 2014;80(10):2966-72.

21. Lee JY, Sung BH, Yu BJ, Lee JH, Lee SH, Kim MS, et al. Phenotypic engineering by reprogramming gene transcription using novel artificial transcription factors in Escherichia coli. Nucleic Acids Res. 2008;36(16):102-11.

22. Park KS, Lee DK, Lee H, Lee Y, Jang YS, Kim YH, et al. Phenotypic alteration of eukaryotic cells using randomized libraries of artificial transcription factors. Nat Biotechnol. 2003;21(10):1208-14.

23. van Tol N, van der Zaal BJ. Artificial transcription factor-mediated regulation of gene expression. Plant Sci. 2014;225:58-67.

24. Alper H, Stephanopoulos G. Global transcription machinery engineering: a new approach for improving cellular phenotype. Metab Eng. 2007;9(3):258-67.

25. Klein-Marcuschamer D, Stephanopoulos G. Assessing the potential of mutational strategies to elicit new phenotypes in industrial strains. Proc Natl Acad Sci USA. 2008;105(7):2319-24.

26. Alper H, Moxley J, Nevoigt E, Fink GR, Stephanopoulos G. Engineering yeast transcription machinery for improved ethanol tolerance and production. Science. 2006;314(5805):1565-8.

27. Hong $\mathrm{SH}$, Wang $X$, Wood TK. Controlling biofilm formation, prophage excision and cell death by rewiring global regulator $\mathrm{H}-\mathrm{NS}$ of Escherichia coli. Microb Biotechnol. 2010;3(3):344-56.

28. Hong SH, Lee J, Wood TK. Engineering global regulator Hha of Escherichia coli to control biofilm dispersal. Microb Biotechnol. 2010;3(6):717-28.

29. Chong HQ, Huang L, Yeow JW, Wang I, Zhang HF, Song H, et al. Improving ethanol tolerance of Escherichia coli by rewiring its global regulator CAMP receptor protein (CRP). PLoS One. 2013:8(2):1-9.

30. Zhang $H$, Chong $H$, Ching $C B$, Song $H$, Jiang R. Engineering global transcription factor cyclic AMP receptor protein of Escherichia coli for improved 1-butanol tolerance. Appl Microbiol Biotechnol. 2012:94(4):1107-17.

31. Kovach ME, Elzer PH, Steven Hill D, Robertson GT, Farris MA, Roop RM II, et al. Four new derivatives of the broad-host-range cloning vector pBBR1MCS, carrying different antibiotic-resistance cassettes. Gene. 1995:166:175-6.

32. Conway T, Osman YA, Konnan Jl, Hoffmann EM, Ingram LO. Promoter and nucleotide sequences of the Zymomonas mobilis pyruvate decarboxylase. J Bacteriol. 1987:169(3):949-54.

33. Neale AD, Scopes RK, Kelly JM, Wettenhall REH. The two alcohol dehydrogenases of Zymomonas mobilis purification by differential dye ligand chromatography, molecular characterisation and physiological roles. Eur J Biochem. 1986;154:119-24.

34. Mackenzie KF, Eddy CK, Ingram LO. Modulation of alcohol dehydrogenase isoenzyme levels in Zymomonas mobilis by iron and zinc. J Bacteriol. 1989;171(2):1063-8.

35. $\mathrm{NCBI}$ conserved domain. Search for conserved domains within a protein or coding nucleotide sequence. 2015. http://www.ncbi.nlm.nih.gov/ Structure/cdd/wrpsb.cgi?SEQUENCE $=56544093 \&$ FULL.

36. Seo JS, Chong H, Park HS, Yoon KO, Jung C, Kim JJ, et al. The genome sequence of the ethanologenic bacterium Zymomonas mobilis ZM4. Nat Biotechnol. 2005;23(1):63-8.

37. Palonen $E$, Lindström $M$, Somervuo $P$, Korkeala $H$. Alternative sigma factor $\sigma^{\mathrm{E}}$ has an important role in stress tolerance of Yersinia pseudotuberculosis IP32953. Appl Environ Microbiol. 2013;79(19):5970-7. 